Journal of Mathematical Physics, Analysis, Geometry

2015, vol. 11, No. 3, pp. 245-266

\title{
Note on Lieb-Thirring Type Inequalities for a Complex Perturbation of Fractional Laplacian
}

\author{
C. Dubuisson \\ Institut de Mathématiques de Bordeaux Université de Bordeaux 351, cours de la Libération \\ F-33405 Talence cedex \\ E-mail: clement.dubuisson@math.u-bordeaux.fr
}

Received October 24, 2014, revised May 14, 2015

For $s>0$, let $H_{0}=(-\Delta)^{s}$ be the fractional Laplacian. In this paper, we obtain Lieb-Thirring type inequalities for the fractional Schrödinger operator defined as $H=H_{0}+V$, where $V \in L^{p}\left(\mathbb{R}^{d}\right), p \geq 1, d \geq 1$, is a complex-valued potential. Our methods are based on the results of articles by Borichev-Golinskii-Kupin [BGK09] and Hansmann [Han11].

Key words: fractional Schrödinger operator, complex perturbation, discrete spectrum, Lieb-Thirring type inequality.

Mathematics Subject Classification 2010: 35P15 (primary); 30C35, 47A75, 47B10 (secondary).

\section{Introduction}

The article by Abramov-Aslanyan-Davies [AAD01] gave rise to many papers devoted to the study of eigenvalues for complex perturbations of various self-adjoint operators. In recent years, the fractional Laplacian $(-\Delta)^{s}, s>0$, has received an increasing interest due to its numerous applications in applied mathematics and physics (see [DPV12] for references).

For $s>0$, the fractional Laplacian $(-\Delta)^{s}$ is defined with the help of the functional calculus applied to the nonnegative self-adjoint operator $-\Delta$. That is, $(-\Delta)^{s}$ is essentially self-adjoint on $\mathcal{C}_{c}^{\infty}\left(\mathbb{R}^{d} ; \mathbb{C}\right)$, and the domain of its closure is the fractional Sobolev space

$$
W^{s, 2}\left(\mathbb{R}^{d}, \mathbb{C}\right):=\left\{f \in L^{2}\left(\mathbb{R}^{d}\right), \int_{\mathbb{R}^{d}}\left(1+|\zeta|^{2 s}\right)|\widehat{f}(\zeta)|^{2} d \zeta<+\infty\right\}
$$

where $\widehat{f}$ is the Fourier transform of $f$ (see [DPV12, Sec. 3.1]). By the spectral mapping theorem, the spectrum of $(-\Delta)^{s}$ is $\mathbb{R}^{+}=[0 ;+\infty[$.

(C) C. Dubuisson, 2015 
We consider the fractional Schrödinger operator

$$
H=(-\Delta)^{s}+V,
$$

where $V$ is the operator of multiplication by the complex-valued function $V$, and we note $H_{0}:=(-\Delta)^{s}$. In particular, the perturbed operator $H$ is not supposed to be self-adjoint. We assume that $V$ is a relatively compact perturbation of $H_{0}$, i.e., $\operatorname{dom}\left(H_{0}\right) \subset \operatorname{dom}(V)$ and $V\left(\lambda-H_{0}\right)^{-1}$ is compact for $\lambda \in \mathbb{C} \backslash \sigma\left(H_{0}\right)$. The spectrum, the essential spectrum, and the discrete spectrum of $H$ will be denoted by $\sigma(H), \sigma_{\text {ess }}(H)$, and $\sigma_{d}(H)$, respectively. Here, the discrete spectrum is the set of all eigenvalues which are discrete points of the spectrum whose corresponding eigenspaces (or rootspaces) are finite dimensional. Throughout the paper, eigenvalues are counted according to their algebraic multiplicity. The essential spectrum of $H$ is defined by $\sigma_{\mathrm{ess}}(H)=\{\lambda \in \mathbb{C}, \lambda-H$ is not Fredholm $\}$, where a closed operator is a Fredholm operator if it has a closed range and both its kernel and cokernel are finite dimensional. In our situation, $\sigma_{\text {ess }}(H):=$ $\sigma(H) \backslash \sigma_{d}(H)$. For more details on these definitions, see [EE89, Chapter IX]. By Weyl's theorem on essential spectrum (see [RS78, Theorem XIII.14]), we have

$$
\sigma_{\text {ess }}(H)=\sigma_{\text {ess }}\left(H_{0}\right)=\sigma\left(H_{0}\right)=\mathbb{R}^{+},
$$

and the only possible accumulation points of $\sigma_{d}(H)$ lie on $\sigma_{\text {ess }}(H)$.

Our interest in the present topic was motivated by the article of Frank-LiebSeiringer [FLS08] on Hardy-Lieb-Thirring inequalities for fractional Schrödinger operator with real-valued potential $V$. As an application, the authors give a proof of the stability of relativistic matter. In particular, for $0<s<\min \left\{1 ; \frac{d}{2}\right\}, \gamma>0$, and $V_{-} \in L^{\gamma+d / 2 s}\left(\mathbb{R}^{d}\right)$, formula (5.11) in [FLS08] says that

$$
\sum_{\lambda \in \sigma_{d}(H)}|\lambda|^{\gamma} \leq C_{d, s, \gamma} \cdot\left\|V_{-}\right\|_{L^{\gamma+d / 2 s}}^{\gamma+d / 2 s},
$$

where $V_{-}=\max \{0 ;-V\}$ and $C_{d, s, \gamma}$ is defined at [FLS08, formula (5.11)].

In this paper, we obtain Lieb-Thirring type inequalities for the fractional Schrödinger operator $H$ with complex-valued $V$. These inequalities give information on the rate of convergence of points from the discrete spectrum $\sigma_{d}(H)$ to the essential spectrum of $H$. The pertaining references on the subject are [FLLS06], [HS02], and [DHK13].

We will assume a little more than $V$ being a relatively compact perturbation of $H_{0}$. Actually, we will suppose that $V$ is a relatively Schatten-von Neumann perturbation of the fractional Laplacian. Namely, let $\mathbf{S}_{p}, p \geq 1$, be the Schattenvon Neumann class of compact operators (see Section 2.3. for further references on the subject). Saying that the potential $V$ defined on $\mathbb{R}^{d}$ is a relatively Schattenvon Neumann perturbation of $H_{0}$ means that $\operatorname{dom}\left(H_{0}\right) \subset \operatorname{dom}(V)$ and

$$
V\left(\lambda-H_{0}\right)^{-1} \in \mathbf{S}_{p}
$$


for one (and hence for all) $\lambda \in \mathbb{C} \backslash \sigma\left(H_{0}\right)$. Hypothesis (1.3) is fulfilled provided $V \in L^{p}\left(\mathbb{R}^{d}\right)$ and $p>\max \left\{1 ; \frac{d}{2 s}\right\}$ (see Proposition 2.3).

We denote by $d(z, \Omega):=\inf _{w \in \Omega}|z-w|$ the distance between $z \in \mathbb{C}$ and $\Omega \subset \mathbb{C}$. As usual, $x_{+}=\max \{0 ; x\}$. The main results of the present article are the following theorems. The constants $\omega$ and $C_{\omega}$ are defined in (4.1).

Theorem 1.1. Let $H$ be the fractional Schrödinger operator defined by (1.1) with $0<s \leq \frac{d}{2}$ and $V \in L^{p}\left(\mathbb{R}^{d}\right)$ with $p>\frac{d}{2 s}$. Then, for $\tau>0$ small enough, we have

$$
\sum_{\lambda \in \sigma_{d}(H)} \frac{d\left(\lambda, \sigma\left(H_{0}\right)\right)^{p+\tau}}{|\lambda|^{\alpha}(1+|\lambda|)^{\beta}} \leq K \frac{C_{\omega}^{p} \omega^{\beta-\tau}}{\tau} \cdot\|V\|_{L^{p}}^{p},
$$

where the powers are

(1) $\alpha=\min \left\{\frac{p+\tau}{2} ; \frac{d}{2 s}\right\}$,

(2) $\beta=2 \tau+\frac{1}{2}\left(\frac{d}{s}-p-\tau\right)_{+}$.

The constant $K$ depends on $d, p, s$, and $\tau$.

Theorem 1.2. Let $H$ be the fractional Schrödinger operator defined by (1.1) with $s>\frac{d}{2}$ and $V \in L^{p}\left(\mathbb{R}^{d}\right)$ with $p>1$. Then, for $\tau>0$ small enough, we have

$$
\sum_{\lambda \in \sigma_{d}(H)} \frac{d\left(\lambda, \sigma\left(H_{0}\right)\right)^{p+1-\frac{d}{2 s}+\tau}}{|\lambda|^{\alpha}(1+|\lambda|)^{\beta}} \leq K^{\prime} \frac{C_{\omega}^{p} \omega^{\beta-\tau}}{\tau}\|V\|_{L^{p}}^{p}
$$

where the powers are

(1) $\alpha=\frac{1}{2}+\frac{1}{2} \min \left\{p-\frac{d}{2 s}+\tau ; 1\right\}$,

(2) $\beta=2 \tau+\frac{1}{2}\left(\frac{d}{2 s}-p+1-\tau\right)_{+}$.

The constant $K^{\prime}$ depends on $d, p, s$, and $\tau$.

The above theorems essentially rely on complex analysis methods presented in [BGK09], while Theorem 1.3 is based on results of [Han11], obtained with the help of tools of functional analysis and operator theory.

Theorem 1.3. Let $H$ be the fractional Schrödinger operator defined in (1.1) with $s>0$ and $V \in L^{p}\left(\mathbb{R}^{d}\right)$, with $p>\max \left\{1 ; \frac{d}{2 s}\right\}$. Then, for $\tau>0$, the following inequality holds:

$$
\sum_{\lambda \in \sigma_{d}(H)} \frac{d\left(\lambda, \sigma\left(H_{0}\right)\right)^{p}}{(1+|\lambda|)^{\frac{d}{2 s}+\tau}} \leq K^{\prime \prime} \frac{C_{\omega}^{p} \omega^{\frac{d}{2 s}}}{\tau} \cdot\|V\|_{L^{p}}^{p}
$$

with $K^{\prime \prime}$ depending on $d, p, s$, and $\tau$. 
Let us assume that $V$ is real-valued and $V \in L^{p}\left(\mathbb{R}^{d}\right)$ for $p>\max \left\{1 ; \frac{d}{2 s}\right\}$. Then $H=(-\Delta)^{s}+V$ is a self-adjoint operator and $\sigma_{d}(H)$ lies on the negative real half-axis.

In [FLS08], the values of the parameter $s$ are restricted to the range $0<s<$ $\min \left\{1, \frac{d}{2}\right\}$ due to the presence of the magnetic potential (see [FLS08, Sec. 2.1]). Setting $\gamma=p-\frac{d}{2 s}>0,(1.2)$ becomes

$$
\sum_{\lambda \in \sigma_{d}(H)}|\lambda|^{p-\frac{d}{2 s}} \leq C_{p, d, s}\left\|V_{-}\right\|_{L^{p}}^{p}
$$

where, as always, $V_{-}=\min \{V, 0\}$.

Theorems 1.1 and 1.2 give Lieb-Thirring type inequalities for all positive values of $s$. In particular, for $0<s \leq \frac{d}{2}$, (1.4) becomes

$$
\sum_{\lambda \in \sigma_{d}(H)}|\lambda|^{\max \left\{\frac{p+\tau}{2} ; p-\frac{d}{2 s}+\tau\right\}} \leq C_{d, p, s, \omega}\|V\|_{L^{p}}^{p}
$$

Rather expectedly, we see that (1.8) is slightly weaker than (1.7), but our results apply to a considerably larger class of potentials.

We continue with a few words on the notation. The generic constants will be denoted by $C$, that is, they will be allowed to change from one relation to another. For two positive functions $f, g$ defined on a domain $\Omega$ of the complex plane $\mathbb{C}$, we write $f(\lambda) \approx g(\lambda)$ if there are constants $C_{1}, C_{2}>0$ such that $C_{1} f(\lambda) \leq g(\lambda) \leq$ $C_{2} f(\lambda)$ for all $\lambda \in \Omega$. We write $f(\lambda) \lesssim g(\lambda)(f(\lambda) \gtrsim g(\lambda))$ if there is a positive constant $C$ such that $f(\lambda) \leq C g(\lambda)(f(\lambda) \geq C g(\lambda)$, respectively) for $\lambda \in \Omega$. The choice of the domain $\Omega$ will be clear from the context.

To compare Theorem 1.1 and Theorem 1.3, it is convenient to consider a sequence $\left(\lambda_{n}\right) \subset \sigma_{d}(H)$ which converges to $\lambda \in \sigma_{\text {ess }}(H)$. Recall that $\sigma_{\text {ess }}(H)=$ $\sigma\left(H_{0}\right)$. We give details on the comparison between (1.4) and (1.6), the comparison between (1.5) and (1.6) being similar. Without loss of generality, we assume $d\left(\lambda_{n}, \sigma\left(H_{0}\right)\right) \leq 1$.

In the case $\lambda \in] 0 ;+\infty[,(1.6)$ is better than (1.4).

In the case $\lambda=\infty$, the term in (1.4) becomes $\frac{d\left(\lambda_{n}, \sigma\left(H_{0}\right)\right)^{p+\tau}}{\left|\lambda_{n}\right|^{\frac{d}{2 s}+2 \tau}}$, for $n$ large enough, and (1.6) is again better than (1.4).

When $\lambda=0$, the situation is slightly more complicated. Choose $\tau>0$ small enough to guarantee $\alpha / \tau \geq 1$. Then (1.4) is better than (1.6) provided $\operatorname{Re}\left(\lambda_{n}\right) \leq 0$ or $\left|\operatorname{Im}\left(\lambda_{n}\right)\right|^{\tau} \gtrsim\left|\operatorname{Re}\left(\lambda_{n}\right)\right|^{\alpha}$ for $\operatorname{Re}\left(\lambda_{n}\right)>0$. Inequality (1.6) is better than (1.4) in the opposite case, i.e., when $\operatorname{Re}\left(\lambda_{n}\right)>0$ and $\left|\operatorname{Im}\left(\lambda_{n}\right)\right|^{\tau} \lesssim\left|\operatorname{Re}\left(\lambda_{n}\right)\right|^{\alpha}$.

To sum up, we see that neither Theorem 1.1 nor Theorem 1.3 has an advantage over each other. 
As a concluding remark, we would like to mention that it is possible to consider the complex matrix-valued potential $V$ as in [Dub14a], devoted to the study of non-self-adjoint Dirac operators. Unlike the latter paper, the study of matrixvalued potentials is neither natural nor complicated in the present framework. The only difference with the scalar-valued case will be the presence of the constant $n^{p / 2}$ in (3.1) and (3.2), $n$ being the size of the square matrix giving the matrixvalued potential.

At last, we say a few words on the structure of the paper. We recall some known results and give references in Sec. 2. The key point of the proofs is the bound on the resolvent of $H_{0}$, and it is proved in Sec. 3. In Secs. 4 and 5, we prove Theorems 1.1 and 1.2, respectively. In Sec. 6, we deal with Theorem 1.3.

\section{Preliminaries}

\subsection{Theorem of Borichev-Golinskii-Kupin.}

The following theorem, proved in [BGK09, Theorem 0.2], gives a bound on the distribution of zeros of a holomorphic function on the unit disc $\mathbb{D}:=\{|z|<1\}$ in terms of its growth towards the boundary $\mathbb{T}:=\{|z|=1\}$.

Theorem 2.1. Let $h$ be a holomorphic function on $\mathbb{D}$ with $h(0)=1$. Assume that $h$ satisfies a bound of the form

$$
|h(z)| \leq \exp \left(\frac{K}{(1-|z|)^{\alpha}} \prod_{j=1}^{N} \frac{1}{\left|z-\zeta_{j}\right|^{\beta_{j}}}\right),
$$

where $\left|\zeta_{j}\right|=1$ and $\alpha, \beta_{j} \geq 0, j=1, \ldots, N$.

Then, for any $0<\tau<1$, the zeros of $h$ satisfy the inequality

$$
\sum_{h(z)=0}(1-|z|)^{\alpha+1+\tau} \prod_{j=1}^{N}\left|z-\zeta_{j}\right|^{\left(\beta_{j}-1+\tau\right)+} \leq C \cdot K
$$

where $C$ depends on $\alpha, \beta_{j}, \zeta_{j}$ and $\tau$.

Above, $x_{+}=\max \{x, 0\}$.

\subsection{Conformal maps}

Let $\varphi_{a}$ be a conformal map sending $\mathbb{D}$ to the resolvent set of the operator $H_{0}$, $\rho\left(H_{0}\right)=\mathbb{C} \backslash \mathbb{R}^{+}$. For $a>0$, it is given by the relation

$$
\varphi_{a}: z \mapsto \lambda:=-a\left(\frac{z+1}{z-1}\right)^{2},
$$


and the inverse map going from $\mathbb{C} \backslash \mathbb{R}^{+}$to $\mathbb{D}$ is

$$
\varphi_{a}^{-1}: \lambda \mapsto z:=\frac{\sqrt{\lambda}-\mathrm{i} \sqrt{a}}{\sqrt{\lambda}+\mathrm{i} \sqrt{a}}
$$

Later in the paper, we will have to compare the distance from $\lambda=\varphi_{a}(z)$ to the boundary of $\rho\left(H_{0}\right), \partial \rho\left(H_{0}\right)=\mathbb{R}^{+}$, and the distance from $z$ to $\partial \mathbb{D}=\mathbb{T}$. The results of this kind are called distortion theorems.

Proposition 2.2. (Distortion between $\mathbb{C} \backslash \mathbb{R}^{+}$and $\mathbb{D}$ ). Let, as above, $\lambda=$ $\varphi_{a}(z)$. We have

$$
a \cdot d(z, \mathbb{T}) \frac{|z+1|}{|z-1|^{3}} \leq d\left(\lambda, \mathbb{R}^{+}\right) \leq 8 a \cdot d(z, \mathbb{T}) \frac{|z+1|}{|z-1|^{3}},
$$

and

$$
\frac{\sqrt{a}}{4} \frac{d\left(\lambda, \mathbb{R}^{+}\right)}{\sqrt{|\lambda|}(a+|\lambda|)} \leq d(z, \mathbb{T}) \leq 4 \sqrt{a} \frac{d\left(\lambda, \mathbb{R}^{+}\right)}{\sqrt{|\lambda|}(a+|\lambda|)} .
$$

$\mathrm{P}$ r o o f. The first inequality is a direct application of Koebe distortion theorem [Pom92, Corollary 1.4] to the map $\varphi_{a}$, so the proof is omitted.

For the second one, we have

$$
|z+1|=\frac{2 \sqrt{|\lambda|}}{|\sqrt{\lambda}+\mathrm{i} \sqrt{a}|}, \quad|z-1|=\frac{2 \sqrt{a}}{|\sqrt{\lambda}+\mathrm{i} \sqrt{a}|} .
$$

On the other hand, $|\sqrt{\lambda}+\mathrm{i} \sqrt{a}|^{2}=|\lambda|+a+2 \sqrt{a} \operatorname{Im}(\sqrt{\lambda})$, and, since $\operatorname{Im}(\sqrt{\lambda}) \geq 0$, we obtain

$$
|\lambda|+a \leq|\sqrt{\lambda}+\mathrm{i} \sqrt{a}|^{2} \leq(|\sqrt{\lambda}|+|\sqrt{a}|)^{2} \leq 2(a+|\lambda|) .
$$

Going back to inequalities (2.3), we get (2.4).

\subsection{Schatten classes and determinants}

One can find the definitions and properties of Schatten classes and regularized determinants related to these classes in [DHK09] or [Dub14a]. For detailed discussion and proofs, see the monographs by Gohberg-Krein [GK69] and Simon [Sim77].

Let us consider the operator

$$
F(\lambda):=(\lambda+a)(a+H)^{-1} V\left(\lambda-H_{0}\right)^{-1},
$$


where $a$ is large enough to guarantee that $(a+H)$ is invertible. The coming Proposition 2.3 implies that $V\left(\lambda-H_{0}\right)^{-1} \in \mathbf{S}_{p}$ for $\lambda \in \rho\left(H_{0}\right)$, provided $V \in$ $L^{p}\left(\mathbb{R}^{d}\right)$ and $p>\max \left\{1 ; \frac{d}{2 s}\right\}$.

For $\lambda \in \rho\left(H_{0}\right)$, we have $F(\lambda) \in \mathbf{S}_{p}$ and $F$ holomorphic in $\rho\left(H_{0}\right)$, therefore the holomorphic function of interest is, for all $\lambda \in \rho\left(H_{0}\right)$,

$$
f(\lambda):=\operatorname{det}_{\lceil p\rceil}(\operatorname{Id}-F(\lambda)),
$$

where $\lceil p\rceil=\min \{n \in \mathbb{N}, n \geq p\}$.

In particular, the zeros of $f$ are the eigenvalues of $H$ (counted with algebraic multiplicities), and, for $A \in \mathbf{S}_{p}$, we have

$$
\left|\operatorname{det}_{\lceil p\rceil}(\operatorname{Id}-A)\right| \leq \exp \left(\Gamma_{p}\|A\|_{\mathbf{S}_{p}}^{p}\right) .
$$

We also use the well-known inequality from [Sim05, Theorem 4.1], which we call Birman-Solomyak inequality; some authors prefer to call it Kato-SeilerSimon inequality. Observe that this inequality holds true for $1<p \leq 2$ by duality of the case $p \geq 2$.

Proposition 2.3. Let $V \in L^{p}\left(\mathbb{R}^{d}\right)$ be complex-valued with $p>\max \left\{1 ; \frac{d}{2 s}\right\}$, and $s>0$. Assume that $\lambda \in \rho\left(H_{0}\right)$.

Then $V\left(\lambda-H_{0}\right)^{-1} \in S_{p}$, and

$$
\left\|V\left(\lambda-H_{0}\right)^{-1}\right\|_{S_{p}}^{p} \leq(2 \pi)^{-d}\|V\|_{L^{p}}^{p} \cdot\left\|\left(\lambda-|\cdot|^{2 s}\right)^{-1}\right\|_{L^{p}}^{p}
$$

\section{Bound on the Resolvent}

In this section, we bound the expression $\left\|\left(\lambda-|\cdot|{ }^{2 s}\right)^{-1}\right\|_{L^{p}}$ appearing in Proposition 2.3. The difficulty is to obtain the "right" bound when $s>\frac{d}{2}$. Indeed, when $0<s \leq \frac{d}{2}$, we simply adapt the proof from [DHK09] to the dimensions $d \geq 1$. The proof of the bound when $s>\frac{d}{2}$ requires more work.

We will repeatedly use the following elementary inequalities.

\section{Lemma 3.1.}

(1) Let $a, b \geq 0$ and $\alpha>0$, then

$$
\min \left\{1 ; 2^{\alpha-1}\right\}\left(a^{\alpha}+b^{\alpha}\right) \leq(a+b)^{\alpha} \leq \max \left\{1 ; 2^{\alpha-1}\right\}\left(a^{\alpha}+b^{\alpha}\right) .
$$

(2) In particular, with $\alpha=2$, for $a, b \geq 0$, we have

$$
\sqrt{a^{2}+b^{2}} \leq a+b \leq \sqrt{2} \sqrt{a^{2}+b^{2}}
$$

We recall that $\mathrm{v}_{d-1}=\frac{2 \pi^{\frac{d-1}{2}}}{\Gamma\left(\frac{d-1}{2}\right)}$ for $d \geq 2$ and it is convenient to put $\mathrm{v}_{0}=2$ for $d=1$. 
Proposition 3.2. Let $\lambda=\lambda_{0}+\mathrm{i} \lambda_{1} \in \mathbb{C} \backslash \mathbb{R}^{+}$. Set $\delta=\frac{d}{2 s}-1$.

For $0<s \leq \frac{d}{2}$ and $p>\frac{d}{2 s}$, we have

$$
\left\|\left(\lambda-|\cdot|^{2 s}\right)^{-1}\right\|_{L^{p}}^{p} \leq \frac{\mathrm{v}_{d-1}}{2 s} M_{1} \frac{|\lambda|^{\frac{d}{2 s}}-1}{d\left(\lambda, \sigma\left(H_{0}\right)\right)^{p-1}},
$$

where $M_{1}=\max \left\{K_{2} ; \int_{0}^{+\infty} \frac{t^{\delta} d t}{\left(t^{2}+1\right)^{\frac{p}{2}}}\right\}$, and $K_{2}$ is defined in (3.7) and depends on $d, p$, and $s$.

For $s>\frac{d}{2}$ and $p>1$, we have

$$
\left\|\left(\lambda-|\cdot|^{2 s}\right)^{-1}\right\|_{L^{p}}^{p} \leq \frac{\mathrm{v}_{d-1}}{2 s} \frac{N_{1}}{d\left(\lambda, \sigma\left(H_{0}\right)\right)^{p-\frac{d}{2 s}}},
$$

where $N_{1}=\max \left\{\int_{0}^{+\infty} \frac{t^{\delta}}{\left(t^{2}+1\right)^{\frac{p}{2}}} d t ; \int_{0}^{1} t^{\delta} d t+2 \int_{0}^{+\infty} \frac{d t}{\left(t^{2}+1\right)^{\frac{p}{2}}}\right\}$.

$\mathrm{P}$ r o o f. We start with the polar change of variables

$$
\left\|\left(\lambda-|x|^{2 s}\right)^{-1}\right\|_{L^{p}}^{p}=\mathrm{v}_{d-1} \int_{0}^{+\infty} \frac{r^{d-1}}{\left|r^{2 s}-\lambda\right|^{p}} d r
$$

and we put

$$
I=\int_{0}^{+\infty} \frac{r^{d-1}}{\left|r^{2 s}-\lambda\right|^{p}} d r=\int_{0}^{+\infty} \frac{r^{d-1}}{\left|\left(r^{2 s}-\lambda_{0}\right)^{2}+\lambda_{1}^{2}\right|^{\frac{p}{2}}} d r
$$

First, we assume that $\lambda_{0}<0$, that is, $d\left(\lambda, \sigma\left(H_{0}\right)\right)=|\lambda|$. In (3.3), we use $\left(r^{2 s}-\lambda_{0}\right)^{2} \geq r^{4 s}+\lambda_{0}^{2}$, and we make the change of variables $t=\frac{r^{2 s}}{|\lambda|}$, so

$$
I \leq \int_{0}^{+\infty} \frac{r^{d-1}}{\left(r^{4 s}+|\lambda|^{2}\right)^{\frac{p}{2}}} d r=\frac{1}{2 s} \frac{|\lambda|^{\frac{d}{2 s}}}{|\lambda|^{p}} \int_{0}^{+\infty} \frac{t^{\frac{d}{2 s}}-1}{\left(t^{2}+1\right)^{\frac{p}{2}}} d t .
$$

The integrals in (3.4) converge since $p>\frac{d}{2 s}>0$. Hence, for $\lambda_{0}<0$,

$$
I \leq \frac{1}{2 s} \frac{|\lambda|^{\frac{d}{2 s}-1}}{d\left(\lambda, \sigma\left(H_{0}\right)\right)^{p-1}} \int_{0}^{+\infty} \frac{t^{\frac{d}{2 s}-1}}{\left(t^{2}+1\right)^{\frac{p}{2}}} d t .
$$


Second, we assume $\lambda_{0} \geq 0$ and $\lambda_{1}>0$ (since $\left.\left\|\left(\lambda-|x|^{2 s}\right)^{-1}\right\|=\left\|\left(\bar{\lambda}-|x|^{2 s}\right)^{-1}\right\|\right)$. In (3.3), with the help of the change of variables $t=\frac{r^{2 s}-\lambda_{0}}{\lambda_{1}}$ we obtain

$$
\begin{aligned}
I & =\frac{1}{2 s \lambda_{1}^{p-1}} \int_{-\frac{\lambda_{0}}{\lambda_{1}}}^{+\infty} \frac{\left(\lambda_{1} t+\lambda_{0}\right)^{\frac{d-1}{2 s}}\left(\lambda_{1} t+\lambda_{0}\right)^{\frac{1}{2 s}-1}}{\left(t^{2}+1\right)^{\frac{p}{2}}} d t \\
& =\frac{1}{2 s \lambda_{1}^{p-1}} \int_{-\frac{\lambda_{0}}{\lambda_{1}}}^{+\infty} \frac{\left(\lambda_{1} t+\lambda_{0}\right)^{\frac{d}{2 s}}-1}{\left(t^{2}+1\right)^{\frac{p}{2}}} d t .
\end{aligned}
$$

We distinguish two cases: $0<s \leq \frac{d}{2}$ and $s>\frac{d}{2}$.

If $s=\frac{d}{2}$, for $\operatorname{Re}(\lambda) \geq 0$, bound (3.1) is obvious from (3.6).

Now assume that $0<s<\frac{d}{2}$ and put $\delta=\frac{d}{2 s}-1$. Since $\lambda_{1}>0,-\frac{\lambda_{0}}{\lambda_{1}}<0$, we have

$$
\int_{-\frac{\lambda_{0}}{\lambda_{1}}}^{+\infty} \frac{\left(\lambda_{1} t+\lambda_{0}\right)^{\delta}}{\left(t^{2}+1\right)^{\frac{p}{2}}} d t=\int_{-\frac{\lambda_{0}}{\lambda_{1}}}^{0} \frac{\left(\lambda_{1} t+\lambda_{0}\right)^{\delta}}{\left(t^{2}+1\right)^{\frac{p}{2}}} d t+\int_{0}^{+\infty} \frac{\left(\lambda_{1} t+\lambda_{0}\right)^{\delta}}{\left(t^{2}+1\right)^{\frac{p}{2}}} d t .
$$

In the first integral on the right-hand side of this equality, we use that $\lambda_{1} t+$ $\lambda_{0} \leq \lambda_{0}$. As for the second term, we observe that, by Lemma 3.1, $\left(\lambda_{1} t+\lambda_{0}\right)^{\delta} \leq$ $C_{d, s}\left(\left(\lambda_{1} t\right)^{\delta}+\lambda_{0}^{\delta}\right)$. Here, $C_{d, s}=\max \left\{1 ; 2^{\delta-1}\right\}$. Hence, we have

$$
\begin{aligned}
I & \leq \frac{1}{2 s \lambda_{1}^{p-1}}\left[\lambda_{0}^{\delta} \int_{-\frac{\lambda_{0}}{\lambda_{1}}}^{0} \frac{1}{\left(t^{2}+1\right)^{\frac{p}{2}}} d t\right. \\
& \left.+C_{d, s} \lambda_{1}^{\delta} \int_{0}^{+\infty} \frac{t^{\delta}}{\left(t^{2}+1\right)^{\frac{p}{2}}} d t+C_{d, s} \lambda_{0}^{\delta} \int_{0}^{+\infty} \frac{1}{\left(t^{2}+1\right)^{\frac{p}{2}}} d t\right] \\
& \leq \frac{K_{1}}{2 s \lambda_{1}^{p-1}}\left[\lambda_{0}^{\delta}+\lambda_{1}^{\delta}\right]
\end{aligned}
$$

where $K_{1}=\max \left\{\left(1+C_{d, s}\right) \int_{\mathbb{R}^{+}} \frac{1}{\left(t^{2}+1\right)^{\frac{p}{2}}} d t ; C_{d, s} \int_{\mathbb{R}^{+}} \frac{t^{\delta}}{\left(t^{2}+1\right)^{\frac{p}{2}}} d t\right\}$. Then, putting $C_{d, s}^{\prime}=\max \left\{1 ; 2^{1-\delta}\right\}$, we have by Lemma 3.1 ,

$$
\begin{aligned}
I & \leq \frac{K_{1}}{2 s \lambda_{1}^{p-1}} C_{d, s}^{\prime}\left(\lambda_{0}+\lambda_{1}\right)^{\delta} \\
& \leq \frac{K_{1}}{2 s \lambda_{1}^{p-1}} C_{d, s}^{\prime}(\sqrt{2})^{\delta}|\lambda|^{\delta} .
\end{aligned}
$$


Consequently, for $\lambda_{0} \geq 0$,

$$
I \leq \frac{K_{2}}{2 s} \frac{|\lambda|^{\frac{d}{2 s}-1}}{d\left(\lambda, \sigma\left(H_{0}\right)\right)^{p-1}},
$$

where $K_{2}=K_{1} \cdot C_{d, s}^{\prime} \cdot 2^{\delta / 2}$. Recalling (3.5), we obtain inequality (3.1) in the case $s \leq \frac{d}{2}$.

Let us turn now to the case $s>\frac{d}{2}$. Suppose again that $\lambda_{1}>0$. We recall (3.6)

$$
I=\frac{1}{2 s \lambda_{1}^{p-1}} \int_{-\frac{\lambda_{0}}{\lambda_{1}}}^{+\infty} \frac{\left(\lambda_{1} t+\lambda_{0}\right)^{\delta}}{\left(t^{2}+1\right)^{\frac{p}{2}}} d t .
$$

Since $-1<\delta=\frac{d}{2 s}-1<0$, we cannot use the previous bound. Making the change of variables $u=t+\frac{\lambda_{0}}{\lambda_{1}}$, we obtain

$$
\begin{aligned}
\int_{-\frac{\lambda_{0}}{\lambda_{1}}}^{+\infty} \frac{\left(\lambda_{1} t+\lambda_{0}\right)^{\delta}}{\left(t^{2}+1\right)^{\frac{p}{2}}} d t & =\lambda_{1}^{\delta} \int_{-\frac{\lambda_{0}}{\lambda_{1}}}^{+\infty} \frac{\left(t+\frac{\lambda_{0}}{\lambda_{1}}\right)^{\delta}}{\left(t^{2}+1\right)^{\frac{p}{2}}} d t \\
& =\lambda_{1}^{\delta} \int_{0}^{+\infty} \frac{u^{\delta}}{\left(\left(u-\frac{\lambda_{0}}{\lambda_{1}}\right)^{2}+1\right)^{\frac{p}{2}}} d u .
\end{aligned}
$$

The last integral is bounded independently of $\lambda$, i.e.,

$$
\begin{aligned}
\int_{0}^{+\infty} \frac{u^{\delta}}{\left(\left(u-\frac{\lambda_{0}}{\lambda_{1}}\right)^{2}+1\right)^{\frac{p}{2}}} d u & \leq \int_{0}^{1} u^{\delta} d u+\int_{1}^{+\infty} \frac{1}{\left(\left(u-\frac{\lambda_{0}}{\lambda_{1}}\right)^{2}+1\right)^{\frac{p}{2}}} d u \\
& \leq \int_{0}^{1} u^{\delta} d u+\int_{\mathbb{R}} \frac{1}{\left(u^{2}+1\right)^{\frac{p}{2}}} d u .
\end{aligned}
$$

Indeed, in the first inequality, we use $\left(u-\frac{\lambda_{0}}{\lambda_{1}}\right)^{2}+1 \geq 1$ when $0 \leq u \leq 1$, and $u^{\delta} \leq 1$ when $u \geq 1$ (since $\delta<0$ and $p>1$ ). Hence, for $\lambda_{0} \geq 0$,

$$
I \leq \frac{K_{3}}{2 s} \frac{\lambda_{1}^{\delta}}{\lambda_{1}^{p-1}}=\frac{K_{3}}{2 s} \frac{1}{d\left(\lambda, \sigma\left(H_{0}\right)\right)^{p-\frac{d}{2 s}}},
$$

with $K_{3}=\int_{0}^{1} u^{\delta} d u+2 \int_{0}^{+\infty} \frac{d u}{\left(u^{2}+1\right)^{\frac{p}{2}}}$. Recalling (3.5), we finish the proof of $(3.2)$. 


\section{Proof of Theorem 1.1}

where

Reminding (2.7), we have $f(\lambda)=\operatorname{det}_{\lceil p\rceil}(\operatorname{Id}-F(\lambda))$ for $\lambda \in \rho\left(H_{0}\right)=\mathbb{C} \backslash \mathbb{R}^{+}$,

$$
F(\lambda):=(\lambda+a)(a+H)^{-1} V\left(\lambda-H_{0}\right)^{-1} \in \mathbf{S}_{p}, \quad p \geq 1 .
$$

Inequality (2.8) implies that

$$
\log (|f(\lambda)|) \leq \Gamma_{p}\left\|(\lambda+a)(a+H)^{-1} V\left(\lambda-H_{0}\right)^{-1}\right\|_{\mathbf{S}_{p}}^{p} .
$$

From [Han10, Lemma 3.3.4], we know the following bound on $\left\|(-a-H)^{-1}\right\|$ for some $a>0$. Then, there exists $\omega \geq 1$, depending on $d, p, s$, and $V$, such that for any $a \geq \omega$,

$$
\left\|(-a-H)^{-1}\right\| \leq \frac{C_{\omega}}{|\omega-a|}
$$

where $C_{\omega}=\left(1-\left\|V\left(-\omega-H_{0}\right)^{-1}\right\|\right)^{-1}$. By Proposition 2.3, we get the next inequality for $p>1$ and $\lambda \in \mathbb{C} \backslash \mathbb{R}^{+}$,

$$
\log |f(\lambda)| \leq \frac{\Gamma_{p}}{(2 \pi)^{d}} \frac{C_{\omega}^{p}}{|\omega-a|^{p}}|\lambda+a|^{p}\|V\|_{L^{p}}^{p}\left\|\left(\lambda-|\cdot|^{2 s}\right)^{-1}\right\|_{L^{p}}^{p}
$$

Since $0<s \leq \frac{d}{2}$, from $(3.1)$, we have

$$
\log |f(\lambda)| \leq \frac{K_{1} C_{\omega}^{p}}{|\omega-a|^{p}}\|V\|_{L^{p}}^{p} \frac{|\lambda+a|^{p}|\lambda|^{\frac{d}{2 s}-1}}{d\left(\lambda, \sigma\left(H_{0}\right)\right)^{p-1}},
$$

where

$$
K_{1}=\frac{\Gamma_{p}}{(2 \pi)^{d}} \frac{\mathrm{v}_{d-1}}{2 s} M_{1}
$$

and $M_{1}$ is defined in (3.1).

We now transfer the above inequality on $\mathbb{D}$ in order to apply Theorem 2.1. That is, we consider the function $g(z)=f \circ \varphi_{a}(z)$, where $\varphi_{a}$ is defined by (2.2).

It is clearly holomorphic on $\mathbb{D}$. By definition (2.2), we see $|\lambda+a|=\frac{4 a|z|}{|z-1|^{2}}$. So, Proposition 2.2 applied to inequality (4.3) gives

$$
\begin{aligned}
\log |g(z)| & \leq \frac{K_{1} C_{\omega}^{p}}{|\omega-a|^{p}}\|V\|_{L^{p}}^{p} \frac{(4 a)^{p}|z|^{p} a^{\frac{d}{2 s}-1}|z+1|^{\frac{d}{s}-2}|z-1|^{3(p-1)}}{|z-1|^{2 p}|z-1|^{\frac{d}{s}-2} a^{p-1} d(z, \mathbb{T})^{p-1}|z+1|^{p-1}} \\
& \leq \frac{K_{2} a^{\frac{d}{2 s}}}{|\omega-a|^{p}}\|V\|_{L^{p}}^{p} \frac{|z|^{p}}{d(z, \mathbb{T})^{p-1}|z+1|^{p-\frac{d}{s}+1}|z-1|^{\frac{d}{s}-p+1}}
\end{aligned}
$$

with $K_{2}=4^{p} K_{1} C_{\omega}^{p}$. 
Now, by Theorem 2.1, we have for all $\tau>0$,

$$
\sum_{g(z)=0}(1-|z|)^{p+\tau}|z-1|^{\left(\frac{d}{s}-p+\tau\right)+}|z+1|^{\left(p-\frac{d}{s}+\tau\right)+} \leq C \frac{K_{2} a^{\frac{d}{2 s}}}{|\omega-a|^{p}}\|V\|_{L^{p}}^{p},
$$

where $K_{2}$ is defined above and $C$ depends on $d, p, s$, and $\tau$. There are three cases to be considered: Case 1: $\frac{d}{2 s}<p<\frac{d}{s}$, Case 2: $p=\frac{d}{s}$, and Case 3: $p>\frac{d}{s}$.

For each case, we will transfer the relation (4.5) back to $\rho\left(H_{0}\right)=\mathbb{C} \backslash \mathbb{R}^{+}$. Recalling Proposition 2.2, we see

$$
\begin{gathered}
1-|z|=d(z, \mathbb{T}) \geq \frac{\sqrt{a}}{4} \frac{d\left(\lambda, \sigma\left(H_{0}\right)\right)}{|\lambda|^{1 / 2}(a+|\lambda|)} \\
|z+1|^{2} \geq \frac{2|\lambda|}{a+|\lambda|}, \quad \text { and } \quad|z-1|^{2} \geq \frac{2 a}{a+|\lambda|} .
\end{gathered}
$$

Then we will integrate the resulting inequality with respect to $a \in[\omega ;+\infty[$ to get to a sharper bound. This integration follows the idea of Demuth, Hansmann, and Katriel (see [DHK09] or [DHK13].

Since the computations are similar for all above cases, we will give the details of the proof in the first case and present only the main steps in the remaining cases.

4.1. Case 1: $\frac{d}{2 s}<p<\frac{d}{s}$

For $0<\tau<\frac{d}{s}-p$, relation (4.6) becomes

$$
\sum_{g(z)=0}(1-|z|)^{p+\tau}|z-1|^{\frac{d}{s}-p+\tau} \leq C \frac{K_{2} a^{\frac{d}{2 s}}}{|\omega-a|^{p}}\|V\|_{L^{p}}^{p}
$$

We transfer this inequality back to $\rho\left(H_{0}\right)$, i.e.,

$$
(1-|z|)^{p+\tau}|z-1|^{\frac{d}{s}-p+\tau} \geq \frac{a^{\frac{d}{2 s}+\tau}}{2^{\frac{5 p}{2}+\frac{3 \tau}{2}-\frac{d}{2 s}}} \frac{d\left(\lambda, \sigma\left(H_{0}\right)\right)^{p+\tau}}{\mid a+\tau}(\lambda \mid)^{\frac{d}{2 s}+\frac{p}{2}+\frac{3 \tau}{2}},
$$

so

$$
\sum_{\lambda \in \sigma_{d}(H)} \frac{d\left(\lambda, \sigma\left(H_{0}\right)\right)^{p+\tau}}{|\lambda|^{\frac{p+\tau}{2}}(a+|\lambda|)^{\frac{d}{2 s}+\frac{p}{2}+\frac{3 \tau}{2}}} \lesssim a^{-\tau} \frac{K_{2}}{|\omega-a|^{p}} 2^{\frac{5 p}{2}+\frac{3 \tau}{2}-\frac{d}{2 s}}\|V\|_{L^{p}}^{p} .
$$

We can now perform the integration with respect to $a \in[\omega ;+\infty[$. All terms in this relation are positive, so we can permute the sum and the integral by the Fubini theorem. Consequently, we obtain

$$
\sum_{\lambda \in \sigma_{d}(H)} \frac{d\left(\lambda, \sigma\left(H_{0}\right)\right)^{p+\tau}}{|\lambda|^{\frac{p+\tau}{2}}} \int_{\omega}^{+\infty} \frac{|\omega-a|^{p} a^{-1}}{(a+|\lambda|)^{\frac{d}{2 s}+\frac{p}{2}+\frac{3 \tau}{2}}} d a \lesssim \int_{\omega}^{+\infty} \frac{d a}{a^{1+\tau}}\|V\|_{L^{p}}^{p} .
$$


Obviously, $\int_{\omega}^{+\infty} \frac{d a}{a^{1+\tau}}=\frac{1}{\tau \omega^{\tau}}$. In the left-hand side of this relation, we use the bound $a \leq a+|\lambda|$ and we make the change of variables $t=\frac{a-\omega}{|\lambda|+\omega}$. Hence, we come to

$$
\begin{aligned}
\int_{\omega}^{+\infty} \frac{|\omega-a|^{p} a^{-1}}{(a+|\lambda|)^{\frac{d}{2 s}+\frac{p}{2}+\frac{3 \tau}{2}}} d a & \geq \int_{\omega}^{+\infty} \frac{|\omega-a|^{p}}{(a+|\lambda|)^{\frac{d}{2 s}+\frac{p}{2}+1+\frac{3 \tau}{2}}} d a \\
& \geq \frac{(|\lambda|+\omega)^{p+1}}{(|\lambda|+\omega)^{\frac{d}{2 s}+\frac{p}{2}+1+\frac{3 \tau}{2}}} \int_{0}^{+\infty} \frac{t^{p} d t}{(t+1)^{\frac{d}{2 s}+\frac{p}{2}+1+\frac{3 \tau}{2}}}
\end{aligned}
$$

So, for $\frac{d}{2 s}<p<\frac{d}{s}$,

$$
\sum_{\lambda \in \sigma_{d}(H)} \frac{d\left(\lambda, \sigma\left(H_{0}\right)\right)^{p+\tau}}{|\lambda|^{\frac{p+\tau}{2}}(\omega+|\lambda|)^{\frac{d}{2 s}-\frac{p}{2}+\frac{3 \tau}{2}}} \leq C \frac{K_{1} C_{\omega}^{p}}{I_{1} \tau \omega^{\tau}} 2^{\delta_{1}}\|V\|_{L^{p}}^{p}
$$

where $K_{1}$ is defined in (4.4), $C$ is defined in (4.6), $I_{1}=\int_{0}^{+\infty} \frac{t^{p} d t}{(t+1)^{\frac{d}{2 s}+\frac{p}{2}+1+\frac{3 \tau}{2}}}$, and $\delta_{1}=\frac{9 p}{2}+\frac{3 \tau}{2}-\frac{d}{2 s}$.

\subsection{Case 2: $p=\frac{d}{s}$}

Reminding (4.6), we obtain

$$
\sum_{g(z)=0}(1-|z|)^{p+\tau}|z-1|^{\tau}|z+1|^{\tau} \leq C \frac{K_{2} a^{\frac{d}{2 s}}}{|\omega-a|^{p}}\|V\|_{L^{p}}^{p} .
$$

Furthermore, we have

$$
(1-|z|)^{p+\tau}|z-1|^{\tau}|z+1|^{\tau} \geq \frac{a^{\frac{p}{2}+\tau}}{2^{2 p+\tau}} \frac{d\left(\lambda, \sigma_{d}\left(H_{0}\right)\right)^{p+\tau}}{|\lambda|^{\frac{p}{2}}(a+|\lambda|)^{p+2 \tau}},
$$

so, for all $0<\tau<1$,

$$
\sum_{\lambda \in \sigma(H)} \frac{d\left(\lambda, \sigma\left(H_{0}\right)\right)^{p+\tau}}{|\lambda|^{\frac{p}{2}}(a+|\lambda|)^{p+2 \tau}} \lesssim a^{-\tau} \frac{K_{2}}{|\omega-a|^{p}} 2^{2 p+\tau}\|V\|_{L^{p}}^{p} .
$$

After integration, we find

$$
\sum_{\lambda \in \sigma_{d}(H)} \frac{d\left(\lambda, \sigma\left(H_{0}\right)\right)^{p+\tau}}{|\lambda|^{\frac{p}{2}}(\omega+|\lambda|)^{2 \tau}} \leq C \frac{K_{1} C_{\omega}^{p}}{I_{2} \tau \omega^{\tau}} 2^{\delta_{2}}\|V\|_{L^{p}}^{p},
$$


where $K_{1}$ is defined in (4.4), $C$ is defined in (4.6), $I_{2}=\int_{0}^{+\infty} \frac{t^{p}}{(t+1)^{p+1+2 \tau}} d t$, and $\delta_{2}=4 p+\frac{3}{2} \tau$. In (4.12), we used $1 \leq 2^{\tau / 2}$ to obtain a clear statement of $\delta_{j}$ in Remark 4.1.

\subsection{Case 3: $p>\frac{d}{s}$}

For $0<\tau<p-\frac{d}{s}$, relation (4.6) becomes

$$
\sum_{g(z)=0}(1-|z|)^{p+\tau}|z+1|^{p-\frac{d}{s}+\tau} \leq C \frac{K_{2} a^{\frac{d}{2 s}}}{|\omega-a|^{p}}\|V\|_{L^{p}}^{p}
$$

Then, we get

$$
(1-|z|)^{p+\tau}|z+1|^{p-\frac{d}{s}+\tau} \geq \frac{a^{\frac{p+\tau}{2}}}{2^{\frac{3}{2}(p+\tau)+\frac{d}{2 s}}} \frac{d\left(\lambda, \sigma\left(H_{0}\right)\right)^{p+\tau}}{|\lambda|^{\frac{d}{2 s}}(a+|\lambda|)^{\frac{3 p}{2}-\frac{d}{2 s}+\frac{3 \tau}{2}}},
$$

and, for $0<\tau<p-\frac{d}{s}$,

$$
\sum_{\lambda \in \sigma_{d}(H)} \frac{d\left(\lambda, \sigma\left(H_{0}\right)\right)^{p+\tau}}{|\lambda|^{\frac{d}{2 s}}(a+|\lambda|)^{\frac{3}{2}(p+\tau)-\frac{d}{2 s}}} \lesssim a^{\frac{d}{2 s}-\frac{p+\tau}{2}} \frac{K_{2}}{|\omega-a|^{p}} 2^{\frac{3}{2}(p+\tau)+\frac{d}{2 s}}\|V\|_{L^{p}}^{p} .
$$

We integrate the above inequality

$$
\sum_{\lambda \in \sigma_{d}(H)} \frac{d\left(\lambda, \sigma\left(H_{0}\right)\right)^{p+\tau}}{|\lambda|^{\frac{d}{2 s}}} \int_{\omega}^{+\infty} \frac{|\omega-a|^{p} a^{\frac{p+\tau}{2}-\frac{d}{2 s}-1-\tau}}{(a+|\lambda|)^{\frac{3}{2}(p+\tau)-\frac{d}{2 s}}} d a \lesssim \frac{\|V\|_{L^{p}}^{p}}{\tau \omega^{\tau}} .
$$

As before, we do the change of variables $t=\frac{a-\omega}{|\lambda|+\omega}$ and we distinguish two cases: if $\frac{1}{2}\left(p-\frac{d}{s}-2-\tau\right)<0$, we use the bound $[(|\lambda|+\omega) t+\omega] \leq(|\lambda|+\omega)(t+1)$, and if $\frac{1}{2}\left(p-\frac{d}{s}-2-\tau\right) \geq 0$, we apply the bound from below $[(|\lambda|+\omega) t+\omega] \geq(|\lambda|+\omega) t$. We present the case $\frac{1}{2}\left(p-\frac{d}{s}-2-\tau\right) \geq 0$ in details, the other cases are analogous and are omitted. We see

$$
\begin{aligned}
& \int_{\omega}^{+\infty} \frac{|\omega-a|^{p} a^{\frac{p}{2}-\frac{d}{2 s}-1-\frac{\tau}{2}}}{(a+|\lambda|)^{\frac{3}{2}(p+\tau)-\frac{d}{2 s}}} d a \\
= & \frac{(|\lambda|+\omega)^{p+1}}{(|\lambda|+\omega)^{\frac{3}{2}(p+\tau)-\frac{d}{2 s}}} \int_{0}^{+\infty} \frac{t^{p}[(|\lambda|+\omega) t+\omega]^{\frac{p}{2}-\frac{d}{2 s}-1-\frac{\tau}{2}}}{(t+1)^{\frac{3}{2}(p+\tau)-\frac{d}{2 s}}} d t \\
\geq & \frac{(|\lambda|+\omega)^{\frac{p}{2}-\frac{d}{2 s}-1-\frac{\tau}{2}}}{(|\lambda|+\omega)^{\frac{p}{2}-\frac{d}{2 s}-1+\frac{3 \tau}{2}}} \int_{0}^{+\infty} \frac{t^{\frac{3 p}{2}-\frac{d}{2 s}-1-\frac{\tau}{2}}}{(t+1)^{\frac{3}{2}(p+\tau)-\frac{d}{2 s}}} d t .
\end{aligned}
$$


So, if $\frac{1}{2}\left(p-\frac{d}{s}-2-\tau\right) \geq 0$, we obtain

$$
\sum_{\lambda \in \sigma_{d}(H)} \frac{d\left(\lambda, \sigma\left(H_{0}\right)\right)^{p+\tau}}{|\lambda|^{\frac{d}{2 s}}(\omega+|\lambda|)^{2 \tau}} \leq C \frac{K_{1} C_{\omega}^{p}}{I_{3} \tau \omega^{\tau}} 2^{\delta_{3}}\|V\|_{L^{p}}^{p}
$$

where $K_{1}$ is defined in (4.4), $C$ is defined in (4.6), $I_{3}=\int_{0}^{+\infty} \frac{t^{\frac{3 p}{2}-\frac{d}{2 s}-1-\frac{\tau}{2}}}{(t+1)^{\frac{3}{2}(p+\tau)-\frac{d}{2 s}}} d t$, and $\delta_{3}=\frac{7}{2} p+\frac{d}{2 s}+\frac{3}{2} \tau$.

When $\frac{1}{2}\left(p-\frac{d}{s}-2-\tau\right)<0$, we obtain

$$
\sum_{\lambda \in \sigma_{d}(H)} \frac{d\left(\lambda, \sigma\left(H_{0}\right)\right)^{p+\tau}}{|\lambda|^{\frac{d}{2 s}}(\omega+|\lambda|)^{2 \tau}} \leq C \cdot \frac{K_{1} C_{\omega}^{p}}{I_{4} \tau \omega^{\tau}} 2^{\delta_{4}}\|V\|_{L^{p}}^{p}
$$

where $K_{1}$ is defined in (4.4), $C$ is defined in (4.6), $I_{4}=\int_{0}^{+\infty} \frac{t^{p}}{(t+1)^{p+1+2 \tau}} d t$, and $\delta_{4}=\delta_{3}=\frac{7}{2} p+\frac{d}{2 s}+\frac{3}{2} \tau$.

In relations $(4.9),(4.12),(4.15)$, and (4.16), we use the bound $\omega+|\lambda| \leq$ $\omega(1+|\lambda|)$, because $\omega \geq 1$, and we come to inequality (1.4). Thus, the proof in the case $0<s \leq \frac{d}{2}$ is finished.

$\mathrm{R}$ e $\mathrm{m}$ a $\mathrm{r} \mathrm{k}$ 4.1. In the above inequalities, one has

$$
\begin{aligned}
I_{j} & =\int_{0}^{+\infty} \frac{t^{p+\frac{1}{2}\left(p-\frac{d}{s}-2-\tau\right)_{+}}}{(t+1)^{p+1+2 \tau+\frac{1}{2} \max \left\{\frac{d}{s}-p-2 \tau ; 0 ; p-\frac{d}{s}-2-\tau\right\}}} d t, \\
\delta_{j} & =\frac{7 p}{2}+\frac{3 \tau}{2}+\min \left\{p ; \frac{d}{s}\right\}-\frac{d}{2 s},
\end{aligned}
$$

where $j=1, \ldots, 4$.

\section{Proof of Theorem 1.2}

Taking into account (3.2) and (4.1), inequality (4.2) becomes for $\lambda \in \rho\left(H_{0}\right)$,

$$
\log |f(\lambda)| \leq \frac{K_{4} C_{\omega}^{p}}{|\omega-a|^{p}}\|V\|_{L^{p}}^{p} \frac{|\lambda+a|^{p}}{d\left(\lambda, \sigma\left(H_{0}\right)\right)^{p-\frac{d}{2 s}}},
$$

since $s>\frac{d}{2}$ and we have

$$
K_{4}=\frac{\Gamma_{p}}{(2 \pi)^{d}} \frac{\mathrm{v}_{d-1}}{2 s} N_{1}
$$

where $N_{1}$ depends on $d, p$, and $s$ only. As before, we have

$$
\log |g(z)| \leq \frac{K_{4} C_{\omega}^{p}}{|\omega-a|^{p}} \frac{4^{p} a^{\frac{d}{2 s}}|z|^{p}}{d(z, \mathbb{T})^{p-\frac{d}{2 s}}|z-1|^{\frac{3 d}{2 s}-p}|z+1|^{p-\frac{d}{2 s}}} .
$$


We set $K_{5}=4^{p} K_{4} C_{\omega}^{p}$. Applying Theorem 2.1, we have

$$
\begin{aligned}
\sum_{g(z)=0}(1-|z|)^{p-\frac{d}{2 s}+1+\tau}|z-1|^{\left(\frac{3 d}{2 s}-p-1+\tau\right)_{+}} & |z+1|^{\left(p-\frac{d}{2 s}-1+\tau\right)_{+}} \\
\leq & \frac{C K_{5} a^{\frac{d}{2 s}}}{|\omega-a|^{p}}\|V\|_{L^{p}}^{p}
\end{aligned}
$$

The separation in different cases with respect to $p$ and $\frac{d}{2 s}$ is clear from the following picture (Fig. 1). The $x$-axis represents $p$ and the $y$-axis represents $\frac{d}{2 s}$. There are four straight lines given by $y=1, x-y-1=0,-x+3 y-1=0$, and $x-3 y-1=0$.

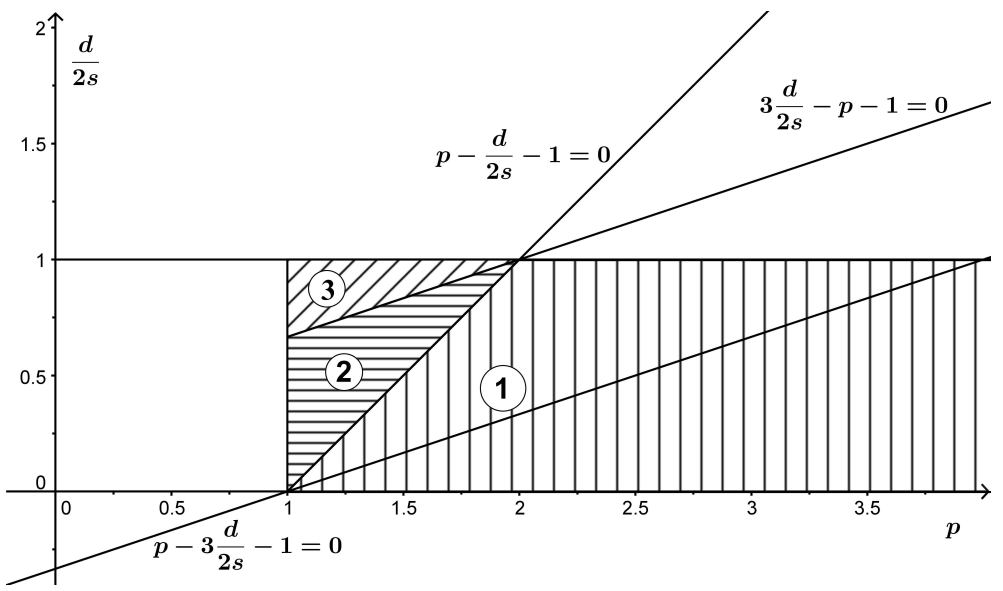

Fig. 1. The different cases

So, we have three different cases to consider: Case 1: $p-\frac{d}{2 s}-1 \geq 0$ and $\frac{3 d}{2 s}-p-1<0$, Case 2: $p-\frac{d}{2 s}-1<0$ and $\frac{3 d}{2 s}-p-1<0$, and Case 3: $p-\frac{d}{2 s}-1<0$ and $\frac{3 d}{2 s}-p-1 \geq 0$. Below, the computations are similar to the case $s \leq \frac{d}{2}$ and thus are omitted.

\subsection{Case 1: $p-\frac{d}{2 s}-1 \geq 0$ and $\frac{3 d}{2 s}-p-1<0$}

We find

$$
\sum_{\lambda \in \sigma_{d}(H)} \frac{d\left(\lambda, \sigma\left(H_{0}\right)\right)^{p-\frac{d}{2 s}+1+\tau}}{|\lambda|(a+|\lambda|)^{3 \frac{p+\tau}{2}-\frac{3 d}{4 s}+\frac{1}{2}}} \lesssim \frac{a^{-\frac{1}{2}\left(p+1-\frac{3 d}{2 s}+\tau\right)}}{|\omega-a|^{p}}\|V\|_{L^{p}}^{p}
$$

It remains to integrate with respect to $a$ on $[\omega ;+\infty[$. We do it in the same way as in the case $0<s \leq \frac{d}{2}$. 
From (5.3), we obtain

$$
\sum_{\lambda \in \sigma_{d}(H)} \frac{d\left(\lambda, \sigma\left(H_{0}\right)\right)^{p+1-\frac{d}{2 s}+\tau}}{|\lambda|} \int_{\omega}^{+\infty} \frac{|\omega-a|^{p} a^{\frac{1}{2}\left(p-\frac{3 d}{2 s}-1-\tau\right)}}{(a+|\lambda|)^{3 \frac{p+\tau}{2}-\frac{3 d}{4 s}+\frac{1}{2}}} d a \lesssim \frac{\|V\|_{L^{p}}^{p}}{\tau \omega^{\tau}} .
$$

Hence, if $p-\frac{3 d}{2 s}-1>0$, and $\tau>0$ is small enough, then

$$
\sum_{\lambda \in \sigma_{d}(H)} \frac{d\left(\lambda, \sigma\left(H_{0}\right)\right)^{p+1-\frac{d}{2 s}+\tau}}{|\lambda|(\omega+|\lambda|)^{2 \tau}} \leq C \frac{K_{4} C_{\omega}^{p}}{I_{5} \tau \omega^{\tau}} 2^{\delta_{5}}\|V\|_{L^{p}}^{p}
$$

where $K_{4}$ is defined in (5.1), $C$ is defined in (5.2), $I_{5}=\int_{0}^{+\infty} \frac{t^{\frac{1}{2}\left(3 p-\frac{3 d}{2 s}-1-\tau\right)}}{(t+1)^{\frac{3 p+\tau}{2}-\frac{3 d}{4 s}+\frac{1}{2}}} d t$, and $\delta_{5}=\frac{1}{2}\left(7 p+5-\frac{3 d}{2 s}+3 \tau\right)$.

Otherwise, if $p-\frac{3 d}{2 s}-1 \leq 0$, we have

$$
\sum_{\lambda \in \sigma_{d}(H)} \frac{d\left(\lambda, \sigma\left(H_{0}\right)\right)^{p+1-\frac{d}{2 s}+\tau}}{|\lambda|(\omega+|\lambda|)^{2 \tau}} \leq C \frac{K_{4} C_{\omega}^{p}}{I_{6} \tau \omega^{\tau}} 2^{\delta_{6}}\|V\|_{L^{p}}^{p}
$$

where $K_{4}$ is defined in (5.1), $C$ is defined in (5.2), $I_{6}=\int_{0}^{+\infty} \frac{t^{p}}{(t+1)^{p+1+2 \tau}} d t$, and $\delta_{6}=\delta_{5}=\frac{1}{2}\left(7 p+5-\frac{3 d}{2 s}+3 \tau\right)$.

5.2. Case 2: $p-\frac{d}{2 s}-1<0$ and $\frac{3 d}{2 s}-p-1<0$

We have

$$
\sum_{\lambda \in \sigma_{d}(H)} \frac{d\left(\lambda, \sigma\left(H_{0}\right)\right)^{p-\frac{d}{2 s}+1+\tau}}{|\lambda|^{\frac{1}{2}\left(p+1-\frac{d}{2 s}+\tau\right)}(a+|\lambda|)^{p+1-\frac{d}{2 s}+\tau}} \lesssim \frac{a^{-\frac{1}{2}\left(p+1-\frac{3 d}{2 s}+\tau\right)}}{|\omega-a|^{p}}\|V\|_{L^{p}}^{p}
$$

Integrating this inequality gives

$$
\sum_{\lambda \in \sigma_{d}(H)} \frac{d\left(\lambda, \sigma\left(H_{0}\right)\right)^{p+1-\frac{d}{2 s}+\tau}}{|\lambda|^{\frac{1}{2}\left(p+1-\frac{d}{2 s}+\tau\right)}(\omega+|\lambda|)^{\frac{1}{2}\left(\frac{d}{2 s}-p+1+3 \tau\right)}} \leq C \frac{K_{4} C_{\omega}^{p}}{I_{7} \tau \omega^{\tau}} 2^{\delta_{7}}\|V\|_{L^{p}}^{p},
$$

where $K_{4}$ is defined in (5.1), $C$ is defined in (5.2), $I_{7}=\int_{0}^{+\infty} \frac{t^{p} d t}{(t+1)^{\frac{1}{2}\left(p+\frac{d}{2 s}+3+3 \tau\right)}}$, and $\delta_{7}=2\left(2 p+1-\frac{d}{2 s}+\tau\right)$. We recall that $0<p-\frac{d}{2 s}<1$, hence $\frac{d}{2 s}-p+1>0$. 
5.3. Case 3: $p-\frac{d}{2 s}-1<0$ and $\frac{3 d}{2 s}-p-1 \geq 0$

This time, we have

$$
\sum_{\lambda \in \sigma_{d}(H)} \frac{d\left(\lambda, \sigma\left(H_{0}\right)\right)^{p-\frac{d}{2 s}+1+\tau}}{|\lambda|^{\frac{p+1+\tau}{2}-\frac{d}{4 s}}(a+|\lambda|)^{\frac{p+1}{2}+\frac{d}{4 s}+\frac{3 \tau}{2}}} \lesssim \frac{a^{-\tau}}{|\omega-a|^{p}}\|V\|_{L^{p}}^{p} .
$$

After integration, the previous inequality becomes

$$
\sum_{\lambda \in \sigma_{d}(H)} \frac{d\left(\lambda, \sigma\left(H_{0}\right)\right)^{p+1-\frac{d}{2 s}+\tau}}{|\lambda|^{\frac{1}{2}\left(p+1-\frac{d}{2 s}+\tau\right)}(\omega+|\lambda|)^{\frac{1}{2}\left(\frac{d}{2 s}-p+1+3 \tau\right)}} \leq C \frac{K_{4} C_{\omega}^{p}}{I_{8} \tau \omega^{\tau}} 2^{\delta_{8}}\|V\|_{L^{p}}^{p},
$$

where $K_{4}$ is defined in (5.1), $C$ in $(5.2), I_{8}=\int_{0}^{+\infty} \frac{t^{p} d t}{(t+1)^{\frac{1}{2}\left(p+3+\frac{d}{2 s}+3 \tau\right)}}$, and $\delta_{8}=\frac{9}{2} p+\frac{5}{2}-\frac{7 d}{4 s}+\frac{3}{2} \tau$. As before, $0<p-\frac{d}{2 s}<1$, and so $\frac{d}{2 s}-p+1>0$.

To make the statement of the theorem more transparent in Case 2, we use $\frac{1}{(1+|\lambda|)^{\tau}} \geq \frac{1}{(1+|\lambda|)^{\frac{3 \tau}{2}}}$. This gives the power $\beta$ in relation (1.5). Finally, since $\omega \geq 1$, we bound $\omega+|\lambda| \leq w(1+|\lambda|)$. The proof of Theorem 1.2 is finished.

$\mathrm{R}$ e $\mathrm{m}$ a $\mathrm{r} \mathrm{k}$ 5.1. In the above inequalities, one has

$$
\begin{aligned}
I_{j} & =\int_{0}^{+\infty} \frac{t^{p+\frac{1}{2}\left(p-\frac{3 d}{2 s}-1-\tau\right)_{+}}}{(t+1)^{p+1+2 \tau+\frac{1}{2} \max \left\{p-\frac{3 d}{2 s}-1-\tau ; 0 ; \frac{d}{2 s}+1-p-\tau\right\}}} d t, \\
\delta_{j} & =2\left(2 p+1-\frac{d}{2 s}+\tau\right)-\frac{1}{2} \max \left\{p-\frac{d}{2 s}-1+\tau ; 0 ; \frac{3 d}{2 s}-p-1+\tau\right\},
\end{aligned}
$$

where $j=5, \ldots, 8$.

\section{Lieb-Thirring Bound Using a Theorem from [Han11]}

\subsection{Hansmann's theorem and conformal mapping}

The following theorem is the key ingredient for the proof of (1.6). It is proved in [Han11].

Theorem 6.1. Let $A$ be a normal bounded operator and $B$ be an operator such that $B-A \in \mathbf{S}_{p}$ for some $p \geq 1$. Suppose also that $\sigma(A)$ is convex. Then the following inequality holds:

$$
\sum_{\lambda \in \sigma_{d}(B)} d(\lambda, \sigma(A))^{p} \leq\|B-A\|_{\mathbf{S}_{p}}^{p} .
$$


Hansmann has another result in this direction (see [Han13, Cor. 1]). Since in our situation the spectrum $\sigma(A)$ is convex, it gives no improvement.

We will apply Theorem 6.1 to $(-a-H)^{-1}$ and $\left(-a-H_{0}\right)^{-1}$, and so we introduce the parameter $a>0$. As in the previous section, we need a distortion result. Introduce a conformal map $g: \mathbb{C} \backslash \mathbb{R}^{+} \rightarrow \overline{\mathbb{C}} \backslash\left[-\frac{1}{a}, 0\right]$ defined by

$$
g(\lambda)=\frac{-1}{a+\lambda}
$$

Below, we denote by $\lambda$ and $\mu$ the variables in $\mathbb{C} \backslash \mathbb{R}^{+}$and $\overline{\mathbb{C}} \backslash\left[-\frac{1}{a}, 0\right]$, respectively.

Proposition 6.2. For $\lambda \in \mathbb{C} \backslash \mathbb{R}^{+}$, we have the bound

$$
d\left(g(\lambda),\left[-\frac{1}{a}, 0\right]\right) \geq \frac{1}{2 \sqrt{5}} \frac{d\left(\lambda, \mathbb{R}^{+}\right)}{(a+|\lambda|)^{2}} .
$$

P r o o f. We obtain a bound for the function $\widetilde{g}: \mathbb{C} \backslash\left[a ;+\infty\left[\rightarrow \overline{\mathbb{C}} \backslash\left[0 ; \frac{1}{a}\right]\right.\right.$ defined by $\widetilde{g}(\lambda)=\frac{1}{\lambda}$ and then compose it by the translation $T: \lambda \mapsto \lambda+a$, that is, $g=-\widetilde{g} \circ T$. After some technical computations (see [Dub14b]), we find

$$
d\left(\widetilde{g}(\lambda),\left[0 ; \frac{1}{a}\right]\right) \geq \frac{1}{\sqrt{5}} \frac{d(\lambda,[a ;+\infty[)}{|\lambda| \cdot(a+|\lambda|)} .
$$

The claimed inequality follows.

As in the proofs of Theorems 1.1 and 1.2, we use an integration with respect to the parameter $a$ to improve the rate of convergence in the left-hand side of inequality (1.6). This trick is borrowed from Theorem 5.3.3 in [DHK13]. We recall that $\omega$ is defined in (4.1).

\subsection{Proof of Theorem 1.3}

We put $A=\left(-a-H_{0}\right)^{-1}$ which is normal and $B=(-a-H)^{-1}$ which is bounded, for $a>\omega$, so that $A$ and $B$ exist. We know that $B-A=B V A \in \mathbf{S}_{p}$, hence we can apply Theorem 6.1 . For $p \geq 1$, it gives

$$
\sum_{\mu \in \sigma_{d}(B)} d(\mu, \sigma(A))^{p} \leq\|B-A\|_{\mathbf{S}_{p}}^{p} .
$$

For $p>\max \left\{1 ; \frac{d}{2 s}\right\}$, we bound the right-hand side of inequality (6.1) with the help of Proposition 2.3 and the inequalities (4.1), (3.5)

$$
\begin{aligned}
\|B-A\|_{\mathbf{S}_{p}}^{p} & \leq(2 \pi)^{-d}\left\|(-a-H)^{-1}\right\|^{p}\|V\|_{L^{p}}^{p}\left\|\left(-a-|x|^{2 s}\right)^{-1}\right\|_{L^{p}}^{p} \\
& \leq K_{1} C_{\omega}^{p} \frac{a^{\frac{d}{2 s}-p}}{|\omega-a|^{p}}\|V\|_{L^{p}}^{p}
\end{aligned}
$$


where

$$
K_{1}=\frac{\mathrm{v}_{d-1}}{2 s(2 \pi)^{d}} \int_{\mathbb{R}^{+}} \frac{t^{\frac{d}{2 s}-1}}{\left(t^{2}+1\right)^{p / 2}} d t .
$$

Then $\mu=(-a-\lambda)^{-1}=g(\lambda) \in \sigma_{d}(B)$ if and only if $\lambda \in \sigma_{d}(H)$, hence

$$
\begin{aligned}
\sum_{\mu \in \sigma_{d}(B)} d(\mu, \sigma(A))^{p} & =\sum_{\left\{g(\lambda), \lambda \in \sigma_{d}(H)\right\}} d(g(\lambda), \sigma(A))^{p} \\
& \geq \frac{1}{(2 \sqrt{5})^{p}} \sum_{\lambda \in \sigma_{d}(H)} \frac{d\left(\lambda, \sigma\left(H_{0}\right)\right)^{p}}{(a+|\lambda|)^{2 p}} .
\end{aligned}
$$

The last inequality results from Proposition 6.2. Thus we obtain

$$
\sum_{\lambda \in \sigma_{d}(H)} \frac{d\left(\lambda, \sigma\left(H_{0}\right)\right)^{p}}{(a+|\lambda|)^{2 p}} \leq(2 \sqrt{5})^{p} K_{1} C_{\omega}^{p} \frac{a^{\frac{d}{2 s}-p}}{|\omega-a|^{p}}\|V\|_{L^{p}}^{p},
$$

where $K_{1}$ is defined in (6.2).

The next step of the proof is the integration with respect to the parameter $a$. Since the computations are similar to the integration performed in Sec. 4, the technical details are omitted. We obtain from (6.3)

$$
\sum_{\lambda \in \sigma_{d}(H)} d\left(\lambda, \sigma\left(H_{0}\right)\right)^{p} \int_{\omega}^{+\infty} \frac{a^{p-\frac{d}{2 s}-1-\tau}|\omega-a|^{p}}{(a+|\lambda|)^{2 p}} d a \lesssim \frac{\|V\|_{L^{p}}^{p}}{\tau \omega^{\tau}} .
$$

Hence, assuming first that $p-d / 2 s>1$, we come to

$$
\int_{\omega}^{+\infty} \frac{a^{p-\frac{d}{2 s}-1-\tau}|a-\omega|^{p} d a}{(a+|\lambda|)^{2 p}} \geq \frac{1}{(|\lambda|+\omega)^{\frac{d}{2 s}}+\tau} \int_{0}^{+\infty} \frac{t^{2 p-\frac{d}{2 s}-1-\tau}}{(t+1)^{2 p}} d t .
$$

When $p-d / 2 s<1$, we have

$$
\int_{\omega}^{+\infty} \frac{a^{p-\frac{d}{2 s}-1-\tau}|a-\omega|^{p} d a}{(a+|\lambda|)^{2 p}} \geq \frac{1}{(|\lambda|+\omega)^{\frac{d}{2 s}+\tau}} \int_{0}^{+\infty} \frac{t^{p} d t}{(t+1)^{p+\frac{d}{2 s}+1+\tau}} .
$$

Hence,

$$
\sum_{\lambda \in \sigma_{d}(H)} \frac{d\left(\lambda, \sigma\left(H_{0}\right)\right)^{p}}{(\omega+|\lambda|)^{\frac{d}{2 s}+\tau}} \leq(2 \sqrt{5})^{p} \frac{K_{1} C_{\omega}^{p}}{I \tau \omega^{\tau}}\|V\|_{L^{p}}^{p},
$$

where $K_{1}$ is defined in (6.2), and

$$
I=\int_{0}^{+\infty} \frac{t^{p+\left(p-\frac{d}{2 s}-1-\tau\right)_{+}}}{(t+1)^{p+\frac{d}{2 s}+1+\tau+\left(p-\frac{d}{2 s}-1-\tau\right)_{+}}} d t .
$$

Using $\omega+|\lambda| \leq \omega(1+|\lambda|)$, we complete the proof of Theorem 1.3. 
Acknowledgment. I thank Stanislas Kupin for his helpful comments on the subject.

\section{References}

[AAD01] A.A. Abramov, A. Aslanyan, and E.B. Davies, Bounds on Complex Eigenvalues and Resonances. - J. Phys. A, Math. Gen. 34 (2001), No. 1, 57-72.

[BGK09] A. Borichev, L. Golinskii, and S. Kupin, A Blaschke-Type Condition and its Application to Complex Jacobi Matrices. - Bull. Lond. Math. Soc. 41 (2009), No. 1, 117-123.

[DHK09] M. Demuth, M. Hansmann, and G. Katriel, On the Discrete Spectrum of Non-Selfadjoint Operators. - J. Funct. Anal. 257 (2009), No. 9, 2742-2759.

[DHK13] M. Demuth, M. Hansmann, and G. Katriel, Eigenvalues of Non-Selfadjoint Operators: a Comparison of Two Approaches. - In: Mathematical Physics, Spectral Theory and Stochastic Analysis, pp. 107-163. Basel: Birkhäuser/Springer, 2013.

[DPV12] E. Di Nezza, G. Palatucci and E. Valdinoci, Hitchhiker's Guide to the Fractional Sobolev Spaces. - Bull. Sci. Math. 136 (2012), No. 5, 521-573.

[Dub14a] C. Dubuisson, On Quantitative Bounds on Eigenvalues of a Complex Perturbation of a Dirac Operator. - Integral Equations Oper. Theory 78 (2014), No. 2, 249-269.

[Dub14b] C. Dubuisson, Study of the Discrete Spectrum of Complex Perturbations of Operators from Mathematical Physics. PhD thesis. University of Bordeaux, 2014, soon available at hal.archives-ouvertes.fr.

[EE89] D.E. Edmunds and W.D. Evans, Spectral Theory and Differential Operators. Oxford, Clarendon Press, paperback ed. edition, 1089.

[FLLS06] R.L. Frank, A. Laptev, E.H. Lieb, and R. Seiringer, Lieb-Thirring Inequalities for Schrödinger Operators with Complex-Valued Potentials. - Lett. Math. Phys. 77 (2006), No. 3, 309-316.

[FLS08] R.L. Frank, E.H. Lieb, and R. Seiringer, Hardy-Lieb-Thirring Inequalities for Fractional Schrödinger Operators. - J. Am. Math. Soc. 21 (2008), No. 4, 925-950.

[GK69] I.C. Gohberg and M.G. Krein, Introduction to the Theory of Linear Nonselfadjoint Operators. Translated from the Russian by A. Feinstein. Translations of Mathematical Monographs, Vol. 18. AMS, Providence, RI, 1969.

[Han10] M. Hansmann, On the Discrete Spectrum of Linear Operators in Hilbert Spaces. PhD thesis, TU Clausthal, 2010.

[Han11] M. Hansmann, An Eigenvalue Estimate and its Application to Non-Selfadjoint Jacobi and Schrödinger Operators. - Lett. Math. Phys. 98 (2011), No. 1, $79-95$. 
[Han13] M. Hansmann, Variation of Discrete Spectra for Non-Selfadjoint Perturbations of Selfadjoint Operators. - Integral Equations Oper. Theory 76 (2013), No. 2, $163-178$.

[HS02] M. Hansmann, Lieb-Thirring Inequalities for Jacobi Matrices. - J. Approx. Theory 118 (2002), No. 1, 106-130.

[Pom92] C. Pommerenke, Boundary Behaviour of Conformal Maps. Berlin, SpringerVerlag, 1992.

[RS78] M. Reed and B. Simon, Methods of Modern Mathematical Physics. IV: Analysis of Operators. Academic Press [Harcourt Brace Jovanovich, Publishers], New York-London, 1978.

[Sim77] B. Simon, Notes on Infinite Determinants of Hilbert Space Operators. - Adv. Math. 24 (1977), 244-273.

[Sim05] B. Simon, Trace Ideals and their Applications. Providence, RI, American Mathematical Society (AMS), 2nd ed., 2005.

266 Journal of Mathematical Physics, Analysis, Geometry, 2015, vol. 11, No. 3 